facilities, as they are the latest versions of the RAI-MDS.

This project is part of the Marie Skłodowska Curie Actions Innovative Training Network H2020-MSCA-ITN, under grant agreement number 813196

\title{
410 - A Systematic Review on Digital Health Interventions (DHIs) supporting the administration of Comprehensive Geriatric Assessments (CGAs) use in long-term and home care settings
}

Author List

Mauricio Molinari Ulate ${ }^{1,2}$, Aysan Mahmoudi ${ }^{1,2}$, Esther Parra-Vidales ${ }^{2}$, Juan-Luis Muñoz-Sánchez ${ }^{4}$, Henriëtte G. van der Roest ${ }^{5}$, and Manuel A.Franco-Martín ${ }^{1,3,4}$

${ }^{1}$ Institute of Biomedical Research of Salamanca, University of Salamanca

${ }^{2}$ Department of Research and Development, INTRAS Foundation

${ }^{3}$ Psychiatric Department, Zamora Healthcare Complex

${ }^{4}$ Psychiatric Department, Hortega University Hospital

${ }^{5}$ Department on Aging, Netherlands Institute of Mental Health and Addiction (Trimbos Institute)

Background:

Health systems are in the need for novel approaches to tackle the challenges from the demographic transition to ageing populations. An effective approach to face these challenges is integrated care delivery. Implementing Comprehensive Geriatric Assessments (CGAs) in Health Ageing might be highly relevant to incorporate this approach. However, this implementation should be supported by Digital Health Interventions (DHIs) in order to reach their full capacity.

Research Objective:

To identify the DHIs facilitating the administration of CGAs used in long-term care and home care settings and provide an insight on their characteristics and stage of maturity and evaluation.

Method:

A search strategy was conducted in PubMed, CINAHL, and Web of Science, targeting studies evaluating the DHIs facilitating the administration of CGAs used in long-term care and home care settings. Studies in English and Spanish and published up to July 26, 2021 were considered.

Preliminary results of the ongoing study:

A total of four papers describing three digital platforms supporting the administration of the CGAs were identified. Information on implementation reported less completion of some sections affecting the CGAs outcomes, assessments mostly being the responsibility of nurses, and missing data related with less quality of care. Limitations and barriers regarding their usability and feasibility were also identified.

Conclusion:

The inclusion of safe data storage, automatic notifications for assessment completion, automatic calculation of final outcomes, and facilitation of multidisciplinary assessments, were identified as key features to enhance the implementation of digital platforms facilitating the administration of CGAs. Nonetheless, information regarding technical features and hardware information of the digital platforms was scarce.

This project is part of the Marie Skłodowska Curie Actions Innovative Training Network H2020-MSCA-ITN, under grant agreement number 813196 